\title{
The Synthesis of the Optimal Reference Image Using Nominal and Hyperordinal Scales
}

\author{
NataliiaYeromina ${ }^{1}$, Sergey Petrov ${ }^{2}$, Nataliia Antonenko ${ }^{3}$, Ihor Vlasov ${ }^{4}$, Viktoriia Kostrytsia ${ }^{5}$, Vadym Korshenko ${ }^{6}$ \\ ${ }^{1}$ Department of Electronic Computers, Kharkiv National University of Radio Electronics, Ukraine, Kharkiv, \\ nataliia.yeromina@nure.ua \\ 2,3Ukrainian Engineering Pedagogics Academy,Ukraine, psv_topol@ukr.net \\ ${ }^{4,5}$ National Defense University of Ukraine named after Ivan Cherniakhovskyi, Ukraine ,igor.vlas1969@gmail.com \\ ${ }^{6}$ Kharkiv National University of Internal Affairs, Ukraine, vkorshenko@ gmail.com
}

\begin{abstract}
The results of use of one-to-one and hypermonotone transformations in order to perform the synthesis of the optimal image, required for the functioning of the correlation-extreme navigation systems (CENS) of mobile robots (MR) are represented in this article. The algorithm of the synthesis of the optimal RI in the hyperordinal scale is suggested.
\end{abstract}

Key words: nominal scale, hyperordinal scale, optimal reference image, algorithms synthesis, navigation systems of mobile robots.

\section{INTRODUCTION}

The navigation of the airborne MR, equipped with CESN, in conditions of the uncertainty, caused by influence of different stochastic factors, requires the search of optimal solution regarding the RI composition as well as regarding the corresponding algorithms. The process of RI composition with respect to the possible change of the data on the MR reference location causes the necessity to find the simple and accurate solutions, which ensure the required indicators of precision of navigation system $[1,2$, 3].

\subsection{Problem analysis}

The results of preparation of the general approach to optimal RI synthesis using the group of the permissible transformations and corresponding scales are represented in the article [4]. The results of use of the strong and ordinal scales during the synthesis of the RI for vehicle CENS are described in the article [5]. Meanwhile, there are no results of use of nominal and hyperordinal scales for the RI synthesis for CENS of MR.

The object of this article is the RI synthesis in the representations in nominal and hyperordinal scales.

\section{MAIN MATERIAL}

2.1 The synthesis of optimal reference image in nominal scale.

We will solve the problem of optimal RI synthesis in the nominal scale using the group of the permissible transformations, based on the construction of reflection $\mathrm{b}: \mathrm{O}^{\prime} \rightarrow \mathrm{R}$, which aligns each zone of the viewing surface with the corresponding type of background brightness, with the help of the following assumptions. According to $[4,5]$ we introduce a vector $\boldsymbol{\pi} \in \mathbf{R}^{\mathbf{N}}$ whose components are equal to digitization of the brightness of zones. Hence, the RI digitalization vector $\boldsymbol{\kappa} \in \mathbf{R}^{\mathrm{M}}$ will be represented with the help of vector $\boldsymbol{\pi}$ as follows:

$$
\kappa=\mathbf{H} \boldsymbol{\pi}
$$

Let us set the population of the possible permitted digitalizations per zone by $C_{\boldsymbol{\pi}} \subset \mathbf{R}^{\mathrm{N}}$, which in general case is defined by the relation $\rho$ in numerical scale [4]. Thus the population of the possible digitalizations for vector $\boldsymbol{\kappa} \in \mathbf{R}^{\mathrm{M}} \quad$ can be defined by $\mathrm{C}_{\boldsymbol{\kappa}}=\left\{\boldsymbol{\kappa} \in \mathbf{R}^{\mathrm{M}} \mid \boldsymbol{\kappa}=\mathbf{H} \boldsymbol{\pi}, \boldsymbol{\pi} \in \mathrm{C}_{\boldsymbol{\pi}}\right\}$.

In weak scales the RI representation is written as:

$$
\widehat{\boldsymbol{\kappa}}=\arg \min _{\mathbf{\kappa} \in \mathrm{C}_{\mathbf{\kappa}}}\|\mathbf{y}-\mathbf{\kappa}\|_{\mathbf{A}}^{2} \text {. }
$$

The problem (2) can be equivalent to the following problem:

$$
\widehat{\boldsymbol{\pi}}=\arg \min _{\pi \in \mathrm{C}_{\boldsymbol{\pi}}}\|\mathbf{y}-\mathbf{H} \boldsymbol{\pi}\|_{\mathbf{A}}^{2} .
$$

and the optimum digitization $\widehat{\boldsymbol{\kappa}}$ is restored along the vector $\widehat{\boldsymbol{\pi}}$ by means of a relation (2).

Given the above let us assume that $\mathrm{R}$ is the equivalence relation on $\mathrm{X}$, which divides the set $\mathrm{X}$ into the zones, the brightness of which is described by the components of the vector $\pi$. Different brightness values must correspond to different zones. Consequently, no limits are applied to the components of vector $\pi$, which means $C_{\pi}=\mathbf{R}^{\mathrm{N}}$.

Therefore, $\mathrm{C}_{\boldsymbol{\kappa}}=\left\{\boldsymbol{\kappa} \in \mathbf{R}^{\mathrm{M}} \mid \boldsymbol{\kappa}=\mathbf{H} \boldsymbol{\pi}, \boldsymbol{\pi} \in \mathbf{R}^{\mathrm{N}}\right\}$ is the $\mathrm{N}$ dimension subspace $\mathbf{R}^{\mathrm{M}}$.

The geometric meaning of finding the optimal digitalization $\widehat{\boldsymbol{\kappa}}$ is to specify the projection of vector $\mathbf{y} \in \mathbf{R}^{\mathrm{M}}$ on the subspace $\mathrm{C}_{\boldsymbol{\kappa}} \subset \mathbf{R}^{\mathrm{M}}$.

It is common practice to prenormalize the vector $\mathbf{y}$ while comparing the vectors $\mathbf{y}$ and $\boldsymbol{\kappa}$, which is $\|\mathbf{y}\|_{\mathbf{A}}=1$. Thus let us set the optimal digitalization $\widehat{\boldsymbol{\kappa}}$ to be the normalized vector. Then the problem (2) can be expressed as:

$$
\hat{\mathbf{\kappa}}=\arg \min _{\mathbf{\kappa} \in \mathrm{C}_{\mathbf{\kappa}} \cap \mathrm{S}_{\mathrm{M}}}\|\mathbf{y}-\mathbf{\kappa}\|_{\mathbf{A}}^{2},
$$


where $\mathrm{S}_{\mathrm{M}}=\left\{\boldsymbol{\kappa} \in \mathbf{R}^{\mathrm{M} \mid}\|\mathbf{\kappa}\|_{\mathbf{A}}^{2}=\mathbf{1}\right\}$ is the unit sphere in $\mathbf{R}^{\mathrm{M}}$. Let us present the equivalent problem (3) as follows:

$$
\widehat{\boldsymbol{\pi}}=\arg \min _{\pi \in \mathrm{C}_{\pi}^{\prime}}\|\mathbf{y}-\mathbf{H} \boldsymbol{\pi}\|_{\mathbf{A}}^{2},
$$

where $C_{\pi}=\left\{\boldsymbol{\pi} \in \mathbf{R}^{\mathrm{N}} \mid\|\mathbf{H} \boldsymbol{\pi}\|_{\mathbf{A}}^{2}=1\right\}$.

Theorem 1. The optimal digitalization $\widehat{\boldsymbol{\kappa}} \in \mathrm{C}_{\mathrm{k}} \cap \mathrm{S}_{\mathrm{M}}$, which is the solution of the problem (4) is defined by the expression:

$$
\widehat{\boldsymbol{\kappa}}=\mathbf{P y} /\|\mathbf{P y}\|
$$

where

$$
\mathbf{P}=\mathbf{H}\left(\mathbf{H}^{\prime} \mathbf{A H}\right)^{-\mathbf{1}} \mathbf{H}^{\prime} \mathbf{A}
$$

- is the matrix of the operator $\mathrm{p}: \mathbf{R}^{\mathrm{M}} \rightarrow \mathrm{C}_{\boldsymbol{k}}$, which is the orthogonal projector on the subspace $\mathrm{C}_{\mathrm{k}}$.

Proof. Let us restate the problem (5) as follows: we will minimize $\mathrm{f}(\boldsymbol{\pi})=\|\mathbf{y}-\mathbf{H} \boldsymbol{\pi}\|_{\mathbf{A}}^{2}$ under restriction

$$
\mathrm{g}(\boldsymbol{\pi})=\|\mathbf{H} \boldsymbol{\pi}\|_{\mathbf{A}}^{2}-1=0,
$$

and compose its Lagrangian

$$
\mathrm{L}(\boldsymbol{\pi}, \mu)=\|\mathbf{y}-\mathbf{H} \boldsymbol{\pi}\|_{\mathbf{A}}^{2}+\mu\left(\|\mathbf{H} \boldsymbol{\pi}\|_{\mathbf{A}}^{2}-1\right) .
$$

The necessary condition for the vector $\hat{\boldsymbol{\pi}}$ to be a solution for the problem (5) is the following:

$$
\nabla_{\pi} \mathrm{L}(\widehat{\boldsymbol{\pi}}, \hat{\mu})=\mathbf{0} .
$$

The direct calculations show us that:

$\nabla_{\pi} f(\pi)=\nabla_{\pi}\left(\|\mathbf{y}-\mathbf{H} \pi\|_{\mathbf{A}}^{2}\right)=2\left(\mathbf{H}^{\prime} \mathbf{A H} \pi-\mathbf{H}^{\prime} \mathbf{A y}\right)$, $\nabla_{\pi} \mathrm{g}(\pi)=\nabla_{\pi}\left(\|\mathbf{H} \pi\|_{\mathbf{A}}^{2}-1\right)=2 \mathbf{H}^{\prime} \mathbf{A H} \pi$.

Then the problem (9) can be expressed as:

$$
(1-\hat{\mu}) \mathbf{H}^{\prime} \mathbf{A H} \widehat{\boldsymbol{\pi}}=\mathbf{H}^{\prime} \mathbf{A y},
$$

based on which

$$
\widehat{\mathbf{\kappa}}=\mathbf{H} \widehat{\boldsymbol{\pi}}=\mathbf{P y} /(1-\hat{\mu}),
$$

where the matrix $\mathbf{P}$ is defined by the expression (7).

Let us substitute $(11)$ into $(8)$ and we will find $(1+\hat{\mu})=$ $\|\mathbf{P y}\|_{\mathrm{A}}$.

Then using (10) we will receive:

$$
\widehat{\boldsymbol{\kappa}}=\frac{\mathbf{P y}}{\|\mathbf{P y}\|_{\mathbf{A}}}, \widehat{\boldsymbol{\pi}}=\frac{\left(\mathbf{H}^{\prime} \mathbf{A H}\right)^{-1} \mathbf{H}^{\prime} \mathbf{A y}}{\|\mathbf{P y}\|_{\mathbf{A}}}
$$

The sufficient condition for $\widehat{\boldsymbol{\pi}}$, defined by the formula (12), to be the solution of the problem (8) is the positive definiteness of the Hessian matrix of second differential coefficients of Lagrangian $\mathrm{L}$, that is the matrix:

$$
\nabla_{\pi}^{2} \mathrm{~L}(\widehat{\boldsymbol{\pi}}, \hat{\mu})=2\|\mathbf{P y}\|_{\mathrm{A}} \mathbf{H}^{\prime} \mathbf{A H} .
$$

It is common knowledge [10] that to obtain the positive definiteness of the matrix it is necessary and sufficient for its principal minors to be positive. The direct calculations show us that:

$$
\mathbf{H}^{\prime} \mathbf{A H}=\operatorname{diag}\left(\mathrm{n}_{1}, \ldots, \mathrm{n}_{\mathrm{N}}\right)
$$

where

$$
\mathrm{n}_{\mathrm{i}}=\sum_{\mathrm{k} \in \mathrm{N}_{\mathrm{i}}} \mathrm{p}_{\mathrm{k}}
$$

Consequently, the matrix $\mathbf{H}^{\prime} \mathbf{A H}$ is scalar and the elements of its main diagonals are positive, therefore the determinant $\operatorname{det} \mathbf{H}^{\prime} \mathbf{A H}>0$ as well as the sufficiency criterion is met.

For the practical calculations of the solution $\hat{\boldsymbol{\pi}}$ it is more convenient to use the resulting expression instead of the matrix representation (12):

$$
\widehat{\pi}_{\mathrm{i}}=\frac{1}{\mathrm{n}_{\mathrm{i}}} \sum_{\mathrm{k} \in \mathrm{N}_{\mathrm{i}}} \mathrm{p}_{\mathrm{k}} \mathrm{y}_{\mathrm{k}}, \mathrm{i} \in \overline{1, \mathrm{~N}},
$$

which follows from the continued equality:

$$
\begin{aligned}
& \mathrm{h}_{\mathrm{ij}}=\delta_{\mathrm{r}_{\mathrm{i}} \mathrm{j}}, \quad \mathrm{i} \in \overline{1, \mathrm{M}}, \mathrm{j} \in \overline{1, \mathrm{~N}} \\
& \mathbf{F}=\mathbf{H}^{\prime} \mathbf{A}, \mathrm{f}_{\mathrm{ij}}=\sum_{\mathrm{k}=1}^{\mathrm{M}} \delta_{\mathrm{r}_{\mathrm{k}} \mathrm{i}} \mathrm{p}_{\mathrm{k}} \delta_{\mathrm{kj}}=\mathrm{p}_{\mathrm{j}} \delta_{\mathrm{r}_{\mathrm{j}} \mathrm{i}}, \mathrm{i} \in \overline{1, \mathrm{M}}, \mathrm{j} \in \overline{1, \mathrm{~N}} \\
& \mathbf{B}=\mathbf{F H}, \mathrm{b}_{\mathrm{ij}}=\sum_{\mathrm{k}=1}^{\mathrm{M}} \delta_{\mathrm{r}_{\mathrm{k}} \mathrm{i}} \mathrm{p}_{\mathrm{k}} \delta_{\mathrm{r}_{\mathrm{k}} \mathrm{j}}=\delta_{\mathrm{ij}} \mathrm{n}_{\mathrm{i}}, \mathrm{i}, \mathrm{j} \in \overline{1, \mathbf{M}} \text {; } \\
& \mathbf{C}=\mathbf{B}^{-1}, \mathrm{c}_{\mathrm{ij}}=\delta_{\mathrm{ij}} \alpha_{\mathrm{i}}, \alpha_{\mathrm{i}}=1 / \mathrm{n}_{\mathrm{i}}, \mathrm{i}, \mathrm{j} \in \overline{1, \mathrm{M}} \text {; } \\
& \mathbf{D}=\mathbf{C H}^{\prime}, \mathrm{d}_{\mathrm{ij}}=\sum_{\mathrm{k}=1}^{\mathrm{M}} \delta_{\mathrm{r}_{\mathrm{j}} \mathrm{k}} \alpha_{\mathrm{i}} \delta_{\mathrm{ik}}=\alpha_{\mathrm{i}} \delta_{\mathrm{r}_{\mathrm{i}} \mathrm{j}}, \mathrm{i} \in \overline{1, \mathrm{M}}, \mathrm{j} \in \overline{1, \mathrm{~N}} \text {; } \\
& \mathbf{E}=\mathbf{D A}, \mathrm{e}_{\mathrm{ij}}=\sum_{\mathrm{k}=1}^{\mathrm{M}} \alpha_{\mathrm{i}} \delta_{\mathrm{r}_{\mathrm{k}} \mathrm{i}} \mathrm{p}_{\mathrm{k}} \delta_{\mathrm{kj}}=\alpha_{\mathrm{i}} \delta_{\mathrm{r}_{\mathrm{j}} \mathrm{i}} \mathrm{p}_{\mathrm{j}}, \mathrm{i} \in \overline{1, \mathrm{M}}, \mathrm{j} \in \overline{1, N} .
\end{aligned}
$$

The optimal digitalization $\widehat{\boldsymbol{\kappa}}$ is defined by the relation $\widehat{\boldsymbol{\kappa}}=\mathbf{H} \widehat{\boldsymbol{\pi}}$ and can be calculated directly:

$$
\widehat{\kappa}_{\mathrm{i}}=\widehat{\pi}_{\mathrm{r}_{\mathrm{i}}}=\sum_{\mathrm{k} \in \mathrm{N}_{\mathrm{r}_{\mathrm{i}}}} \mathrm{p}_{\mathrm{k}} \mathrm{y}_{\mathrm{k}} / \sum_{\mathrm{k} \in \mathrm{N}_{\mathrm{r}_{\mathrm{i}}}} \mathrm{p}_{\mathrm{k}}, \quad \mathrm{i} \in \overline{1, \mathrm{M}}
$$

In case of equal observations $\left(\mathrm{p}_{1}=\cdots=\mathrm{p}_{\mathrm{M}}=1 / \mathrm{M}\right)$, the expression (15) is represented as:

$$
\left(\pi_{\mathrm{i}}\right)_{\mathrm{opt}}=\overline{\mathrm{z}_{1}}=\frac{1}{\mathrm{~m}_{\mathrm{i}}} \sum_{\mathrm{j} \in \mathrm{N}_{\mathrm{i}}} \mathrm{z}_{\mathrm{j}}, \quad \mathrm{i} \in \overline{1, \mathrm{~N}}
$$

and is represented as sampling mean, defined according to the elements of the current image (CI) fragments, the number of which equals to $\mathrm{m}_{\mathrm{i}}$, and whose numbers correspond to the numbers of $\mathrm{i}$-th zone of RI.

Then the relation (15) can be viewed as the generalization of the sampling mean (17) in case of unequal observations. Using the zonal structure of RI, let us transform the decision function of the nominal algorithm

$$
\mathrm{B}\left(\mathrm{k}, \boldsymbol{\pi}^{\mathrm{k}}\right)=\mathrm{f}\left(\boldsymbol{\pi}^{\mathrm{k}}\right)=\left\|\mathbf{y}-\mathbf{H} \boldsymbol{\pi}^{\mathrm{k}}\right\|_{\mathbf{A}}^{2}
$$

into:

$$
\mathrm{B}\left(\mathrm{k}, \boldsymbol{\pi}^{\mathrm{k}}\right)=\sum_{\mathrm{i}=1}^{\mathrm{N}} \sum_{\mathrm{j} \in \mathrm{N}_{\mathrm{i}}} \mathrm{p}_{\mathrm{j}}^{\mathrm{k}}\left(\mathrm{y}_{\mathrm{j}}^{\mathrm{k}}-\pi_{\mathrm{i}}^{\mathrm{k}}\right)^{2}
$$

Substituting the optimal value of RI for k-th CI fragment, which is defined by the expression (15), into the expression (19), we will receive the decision function of the nominal algorithm as follows: 
NataliiaYeromina et al., International Journal of Emerging Trends in Engineering Research, 8(5), May 2020,2080 - 2084

$$
\mathrm{B}_{\mathrm{nom}}(\mathrm{k})=\mathrm{B}\left(\mathrm{k}, \boldsymbol{\pi}_{\mathrm{opt}}^{\mathrm{k}}\right)=\sum_{\mathrm{i}=1}^{\mathrm{N}} \mathrm{n}_{\mathrm{i}}^{\mathrm{k}} \mathrm{D}_{\mathrm{i}}^{\mathrm{k}}, \mathrm{k} \in \overline{1, \mathrm{R}},
$$

where $\quad D_{i}^{k}=\frac{1}{n_{i}^{k}} \sum_{j \in N_{i}} p_{j}^{k}\left(y_{j}^{k}-\overline{y_{1}^{k}}\right)^{2} \quad$ is the sampling variance, calculated with the help of elements of $\mathrm{k}$-th fragment of CI, whose numbers correspond to the numbers of $\mathrm{i}$-th zone of RI.

In case of equal observations $D_{i}^{k}=\frac{1}{m_{i}} \sum_{j \in N_{i}}\left(y_{j}^{k}-\overline{y_{1}^{k}}\right)^{2}$. Hence, let us write down the decision function (20) as follows:

$$
\mathrm{B}_{\mathrm{nom}}(\mathrm{k})=\frac{1}{\mathrm{M}} \sum_{\mathrm{i}=1}^{\mathrm{N}} \mathrm{m}_{\mathrm{i}} \mathrm{D}_{\mathrm{i}}^{\mathrm{k}}, \quad \mathrm{k} \in \overline{1, \mathrm{R}}
$$

The algorithm with such decision function is called classic zoned algorithm.

Thus, it is proven that in regards to effectiveness the nominal and classic zoned algorithms are equal as they have the same decision functions, and the formula (21) can be viewed as the generalization of the decision function of classic zoned algorithm in case of unequal observations.

As a rule, the vector $\mathbf{y}$ when compared with the RI digitalization, is initially centered.

The vector $\mathbf{y} \in \mathbf{R}^{\mathrm{M}}$ is referred to as centeredone to metric A or A-centered vector if

$$
\left(\mathbf{1}_{M}, \mathbf{y}\right)_{A}=\mathbf{1}_{M}^{\prime} \mathbf{A y}=\sum_{j=1}^{M} p_{j} y_{j}=0
$$

It appears that the following clause is valid.

\section{Clause 1.}

If the vector $\mathbf{y}$ in the problem (4) is A-centered, then the optimal digitalization $\widehat{\mathbf{\kappa}}$ is equally $\mathbf{A}$-centered.

Proof.

The continued equality

$$
\begin{aligned}
\mathbf{1}_{M}^{\prime} \mathbf{A} \widehat{\boldsymbol{\kappa}} & =\sum_{i=1}^{\mathrm{M}} \mathrm{p}_{\mathrm{i}} \hat{\kappa}_{\mathrm{i}}=\sum_{\mathrm{i}=1}^{\mathrm{M}} \mathrm{p}_{\mathrm{i}} \widehat{\pi}_{\mathrm{r}_{\mathrm{i}}}=\sum_{\mathrm{i}=1}^{\mathrm{N}} \widehat{\pi}_{\mathrm{i}} \sum_{\mathrm{k} \in \mathrm{N}_{\mathrm{i}}} \mathrm{p}_{\mathrm{k}}= \\
& =\sum_{\mathrm{i}=1}^{\mathrm{N}} \sum_{\mathrm{k} \in \mathrm{N}_{\mathrm{i}}} \mathrm{p}_{\mathrm{k}} \mathrm{y}_{\mathrm{k}}=\sum_{\mathrm{i}=1}^{\mathrm{M}} \mathrm{p}_{\mathrm{k}} \mathrm{y}_{\mathrm{k}}=0,
\end{aligned}
$$

in deriving of which the relations (15) were taken into consideration, implies the affirmation of the clause.

In case of centered and normalized RI and CI fragments, the decision function (19) is written as:

$$
\mathrm{B}\left(\mathrm{k}, \boldsymbol{\pi}^{\mathrm{k}}\right)=2\left(1-\sum_{\mathrm{i}=1}^{\mathrm{N}} \mathrm{m}_{\mathrm{i}}^{\mathrm{k}} \overline{\mathrm{y}_{1}^{\mathrm{k}}} \mathrm{e}_{\mathrm{i}}\right),
$$

and the algorithm is equal to the picking algorithm, and for the nominal algorithm with the optimal RI, the expression (21) is transformed into

$$
\mathrm{B}_{\mathrm{nom}}(\mathrm{k})=1-\sum_{\mathrm{i}=1}^{\mathrm{N}} \mathrm{m}_{\mathrm{i}}^{\mathrm{k}}\left(\overline{\mathrm{y}_{1}^{\mathrm{k}}}\right)^{2} \text {. }
$$

\subsection{The synthesis of optimal reference image in hyperordinal scale}

In the case in question $\mathrm{R}$ is the relation of hyperorder on $\mathrm{X}$

Let us describe the $C_{\pi}$ as follows:

$\mathrm{C}_{\boldsymbol{\pi}}=\left\{\boldsymbol{\pi} \in \mathbf{R}^{\mathrm{N}} \mid \pi_{1} \leq \cdots \leq \pi_{\mathrm{N}} ; \pi_{\mathrm{i}+1}-\pi_{\mathrm{i}} \leq \pi_{\mathrm{i}+2}-\right.$

$\pi_{i+1}$, ifa $_{i}=-1 ; \pi_{i+2}-\pi_{i+1} \leq \pi_{i+1}-\pi_{i}$, if $a_{i}=1$,

(25)

$\mathrm{i} \in \overline{1, \mathrm{~N}-2}$,

where the vector $\mathbf{a}=\left(\mathrm{a}_{1}, \ldots, \mathrm{a}_{\mathrm{N}-2}\right) \in \mathbf{R}^{\mathrm{N}-2}, \mathrm{a}_{\mathrm{i}}= \pm 1$ sets the relation of order on consequent difference components $\pi_{i}, \pi_{i+1}, \pi_{i+2}$.

In this case the problem of definition of optimal digitalization of RI takes the form:

$$
\widehat{\boldsymbol{\kappa}}=\arg \min _{\boldsymbol{\kappa} \in \mathrm{C}_{\boldsymbol{\kappa}}}\|\mathbf{y}-\mathbf{\kappa}\|_{\mathbf{A}}^{2}
$$

and the equivalent problem is written as:

$$
\widehat{\boldsymbol{\pi}}=\arg \min \mathrm{f}(\boldsymbol{\pi})=\arg \min \|\mathbf{y}-\mathbf{H} \boldsymbol{\pi}\|_{\mathbf{A}}^{2}
$$

under restrictions

$$
\mathbf{g}(\boldsymbol{\pi}) \leq \mathbf{0}, \mathbf{h}(\boldsymbol{\pi}) \leq \mathbf{0},
$$

where

$$
\begin{aligned}
& \mathbf{g}(\boldsymbol{\pi}) \in \mathbf{R}^{N-1}, \\
& g_{j}(\boldsymbol{\pi})=\pi_{j}-\pi_{j+1}, \\
& \mathbf{h}(\boldsymbol{\pi}) \in \mathbf{R}^{N-2}, \\
& h_{k}(\boldsymbol{\pi})=a_{k}\left(\pi_{k}+\pi_{k+2}-2 \pi_{k+1}\right), N \geq 3 .
\end{aligned}
$$

Since the function $h(\pi)$ is convex function in consequence of its linearity, the viewed problem is related to the problem type of convex programming. The Lagrange function is written as:

$$
\mathrm{L}(\boldsymbol{\pi}, \boldsymbol{\mu}, \boldsymbol{v})=\mathrm{f}(\boldsymbol{\pi})+\boldsymbol{\mu}^{\prime} \mathrm{g}(\boldsymbol{\pi})+\boldsymbol{v}^{\prime} \mathrm{h}(\boldsymbol{\pi}),
$$

where $\boldsymbol{\mu} \in \mathbf{R}^{\mathrm{N}-1}, \boldsymbol{v} \in \mathbf{R}^{\mathrm{N}-2}$ are the vectors of the undetermined coefficient of Lagrangian.

According to the Kuhn-Tucker theorem [9], if vectors $\boldsymbol{\pi}, \boldsymbol{\mu}, \boldsymbol{v}$ meet the conditions:

$$
\begin{aligned}
& \nabla_{\pi} \mathrm{L}(\boldsymbol{\pi}, \boldsymbol{\mu}, \mathbf{v})=\nabla \mathrm{f}(\boldsymbol{\pi})+\nabla \mathbf{g}(\boldsymbol{\pi}) \boldsymbol{\mu}+\nabla \mathbf{h}(\boldsymbol{\pi}) \mathbf{v}=\mathbf{0} \\
& \mathbf{g}(\boldsymbol{\pi}) \leq \mathbf{0} ; \mathbf{h}(\boldsymbol{\pi}) \leq \mathbf{0} ; \boldsymbol{\mu} \geq \mathbf{0} ; \mathbf{v} \geq \mathbf{0} ; \\
& \mu_{\mathrm{j}} \mathrm{g}_{\mathrm{j}}(\boldsymbol{\pi})=0, \mathrm{j} \in \overline{1, \mathrm{~N}-1} ; v_{\mathrm{k}} \mathrm{h}_{\mathrm{k}}(\boldsymbol{\pi})=0 \\
& \quad \kappa \in \overline{1, \mathrm{~N}-2}
\end{aligned}
$$

Then $\pi$ is the absolute minimum point of the problem (27), (28).

The matrix of the gradient vectors $\mathbf{g}, \mathbf{h}$ have the form: 
NataliiaYeromina et al., International Journal of Emerging Trends in Engineering Research, 8(5), May 2020,2080 - 2084

$$
\begin{aligned}
\nabla \mathbf{g}(\boldsymbol{\pi}) & =\left[\begin{array}{rrrrrr}
1 & 0 & 0 & \cdots & 0 & 0 \\
-1 & 1 & 0 & \cdots & 0 & 0 \\
0 & -1 & 1 & \cdots & 0 & 0 \\
\cdots & \cdots & \cdots & \cdots & \cdots & \cdots \\
0 & 0 & 0 & \cdots & -1 & 1 \\
0 & 0 & 0 & \cdots & 0 & -1
\end{array}\right], \\
\nabla \mathbf{h}(\boldsymbol{\pi}) & =\left[\begin{array}{rrrrrrr}
a_{1} & 0 & 0 & \cdots & 0 & 0 & 0 \\
-2 a_{1} & a_{2} & 0 & \cdots & 0 & 0 & 0 \\
a_{1} & -2 a_{2} & a_{3} & \cdots & 0 & 0 & 0 \\
\cdots & \cdots & \cdots & \cdots & \cdots & \cdots & \cdots \\
0 & 0 & 0 & \cdots & a_{N-4} & -2 a_{N-3} & a_{N-2} \\
0 & 0 & 0 & \cdots & 0 & a_{N-3} & -2 a_{N-2} \\
0 & 0 & 0 & \cdots & 0 & 0 & a_{N-2}
\end{array}\right] .
\end{aligned}
$$

Then the system of equations (30) for definition of vectors $\boldsymbol{\pi}, \boldsymbol{\mu}, \boldsymbol{v}$ is written in the coordinates as follows:

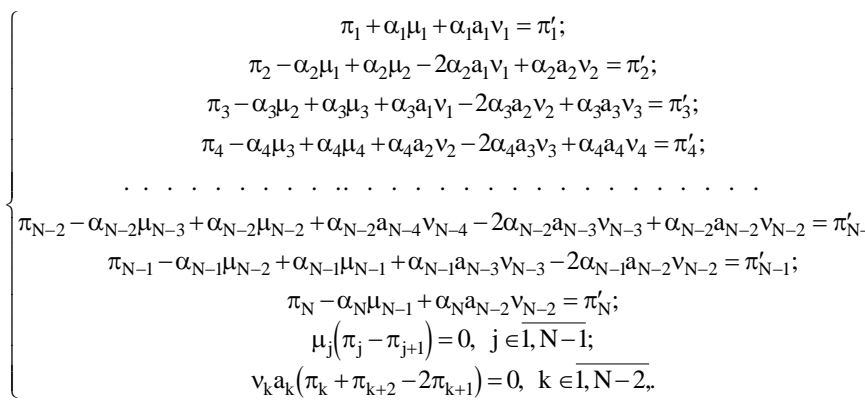

where matrix $\alpha=\operatorname{diag}\left(\alpha_{1}, \ldots, \alpha_{N}\right), \alpha_{i}=1 / 2 n_{i}$.

The matrix of system of equations (31) has the block structure.

\begin{tabular}{r|c|c|c|} 
& $\mathbf{I}_{\mathbf{N}}$ & $\boldsymbol{\alpha} \boldsymbol{\nabla} \mathbf{g}(\boldsymbol{\pi})$ & $\boldsymbol{\alpha} \boldsymbol{\nabla h}(\boldsymbol{\pi})$ \\
\cline { 2 - 4 } $\mathrm{N}-1$ & $\mathbf{M}_{1}$ & $\mathbf{M}_{2}$ & $\mathbf{0}$ \\
\cline { 2 - 4 } $\mathrm{N}-2$ & $\mathbf{M}_{3}$ & $\mathbf{0}$ & $\mathbf{M}_{4}$ \\
\cline { 2 - 4 } & $\mathrm{N}$ & $\mathrm{N}-1$ & $\mathrm{~N}-2$
\end{tabular}

where $\mathbf{I}_{\mathrm{N}}$ is the unity $\mathrm{N} \times \mathrm{N}$-matrix. The blocks $\mathbf{M}_{1}, \mathbf{M}_{2}$ correspond to the system of equations

$\mu_{\mathrm{j}}\left(\pi_{\mathrm{j}}-\pi_{\mathrm{j}+1}\right)=0, \quad \mathrm{j} \in \overline{1, \mathrm{~N}-1}$

and their elements are constructed according to the rule:

if $\pi_{\mathrm{j}}^{\prime}>\pi_{\mathrm{j}+1}^{\prime}$, then $\left(\mathbf{M}_{1}\right)_{\mathrm{ji}}=\left\{\begin{array}{c}1, \mathrm{i}=\mathrm{j}, \\ -1, \mathrm{i}=\mathrm{j}+1, \quad\left(\mathbf{M}_{2}\right)_{\mathrm{ji}}=0, \mathrm{i} \in \overline{1, \mathrm{~N}-1} \\ 0, \mathrm{i} \neq \mathrm{j}, \mathrm{j}+1,\end{array}\right.$

if $\pi_{\mathrm{j}}^{\prime} \leq \pi_{\mathrm{j}+1}^{\prime}$ then $\left(\mathbf{M}_{2}\right)_{\mathrm{ji}}=\delta_{\mathrm{ij}},\left(\mathbf{M}_{1}\right)_{\mathrm{ji}}=0$.

For example, if $\pi_{j}^{\prime} \leq \pi_{j+1}^{\prime}, j \in \overline{1, N-1}$, then $\mathbf{M}_{2}=\mathbf{I}_{\mathrm{N}-1}$, $\mathbf{M}_{1}=0$.

The blocks $\mathbf{M}_{3}, \mathbf{M}_{4}$ correspond to the system of equations:

$$
v_{\mathrm{k}} \mathrm{a}_{\mathrm{k}}\left(\pi_{\mathrm{k}}+\pi_{\mathrm{k}+2}-\pi_{\mathrm{k}+1}\right)=0, \mathrm{k} \in \overline{1, \mathrm{~N}-2} .
$$

Their elements are formed based on the analysis of relations of each triple of consequent components of vector $\boldsymbol{\pi}^{\prime}$ according to the following system of inequalities:

$$
\left\{\begin{array}{c}
\pi_{\mathrm{k}}^{\prime} \leq \pi_{\mathrm{k}+1}^{\prime}\left\{\begin{array}{l}
\pi_{\mathrm{k}-1}^{\prime} \leq \pi_{\mathrm{k}}^{\prime}\left\{\begin{array}{l}
\mathrm{a}_{\mathrm{k}}\left(\pi_{\mathrm{k}}^{\prime}+\pi_{\mathrm{k}+2}^{\prime}-2 \pi_{\mathrm{k}+1}^{\prime}\right) \leq 0 \Rightarrow \delta_{0}, \\
\mathrm{a}_{\mathrm{k}}\left(\pi_{\mathrm{k}}^{\prime}+\pi_{\mathrm{k}+2}^{\prime}-2 \pi_{\mathrm{k}+1}^{\prime}\right)>0 \Rightarrow \delta_{1},
\end{array}\right. \\
\pi_{\mathrm{k}-1}^{\prime}>\pi_{\mathrm{k}}^{\prime} \Rightarrow \delta_{0},
\end{array}\right. \\
\pi_{\mathrm{k}}^{\prime}>\pi_{\mathrm{k}+1}^{\prime} \Rightarrow \delta_{0,}
\end{array}\right.
$$

where $\delta_{0}, \delta_{1}$ are the following rules:

$$
\begin{aligned}
& \delta_{0} \Rightarrow\left(\mathbf{M}_{3}\right)_{\mathrm{kj}}=0,\left(\mathbf{M}_{4}\right)_{\mathrm{kj}}=\delta_{\mathrm{kj}}, \\
& \delta_{1} \Rightarrow\left(\mathbf{M}_{3}\right)_{\mathrm{kj}}=\left\{\begin{array}{c}
-1, \mathrm{j}=\mathrm{k}, \mathrm{k}+2, \\
2, \mathrm{j}=\mathrm{k}+1, \\
0, \mathrm{j} \neq \mathrm{k}, \mathrm{k}+1, \mathrm{k}+2,
\end{array} \quad\left(\mathbf{M}_{4}\right)_{\mathrm{kj}}=0, \mathrm{j} \in \overline{1, \mathrm{~N}-2} .\right.
\end{aligned}
$$

The vectord $\in \mathbf{R}^{3 \mathrm{~N}-3}$ of right parts of the system (30) equals to:

$$
\mathbf{d}=\left(\boldsymbol{\pi}_{1}^{\prime}, \ldots, \boldsymbol{\pi}_{\mathrm{N}}^{\prime}, 0, \ldots, 0\right)
$$

Thus, for optimal digitalization synthesis we can assume ; the following:

Algorithm 1.

Step 1. Repeat the steps $1 . .5$ of the algorithm 1 .

Step 2. Set the vector a.

Step 3. Using the blocked representation (32) of the matrix $\mathbf{M}$ and the relation (33) for the matrix $\nabla \mathbf{g}(\boldsymbol{\pi}), \nabla \mathbf{h}(\boldsymbol{\pi})$, build the first $\mathrm{N}$ lines of the matrix $\mathbf{M}$.

Step 4. Build the next $2 N-3$ lines of matrix $\mathbf{M}$ after the analysis of the system of inequalities (33), (34) and using the rules (35).

Step 5. Build the vector $\mathbf{d} \in \mathbf{R}^{3 \mathrm{~N}-3}$ of the right part of the system (30), using the expression (36).

Step 6. Find the solution $(\boldsymbol{\pi}, \boldsymbol{\mu}, \boldsymbol{v}) \in \mathbf{R}^{3 \mathrm{~N}-3}$ of the system of linear equation (30) using one of the known methods.

Step 7. Find the vector of the optimal digitalization in the relation $\boldsymbol{\kappa}=\mathbf{H} \boldsymbol{\pi}$.

\subsection{The example of the solution of the problem (30)}

As an example, let us take the solution of the problem (30) for $\mathrm{N}=3$.

The cases in question can occur while $\pi_{1}^{\prime} \leq \pi_{2}^{\prime} \leq \pi_{3}^{\prime}$, and (33) implies that $\mu_{1}=\mu_{2}=0$, while the system (30) becomes:

$$
\left\{\begin{array}{c}
\pi_{1}+\alpha_{1} a_{1} v_{1}=\pi_{1}^{\prime} ; \\
\pi_{2}-2 \alpha_{2} a_{1} v_{1}=\pi_{2}^{\prime} ; \\
\pi_{3}+\alpha_{3} a_{1} v_{1}=\pi_{3}^{\prime} ; \\
\mu_{1}=\mu_{2}=0 ; \\
v_{1} a_{1}\left(\pi_{1}+\pi_{3}-2 \pi_{2}\right)=0
\end{array}\right.
$$

Let $a_{1}\left(\pi_{1}+\pi_{3}-2 \pi_{2}\right) \leq 0$. Then, taking into consideration (34), (35) we will receive the trivial solution $\boldsymbol{\pi}=\boldsymbol{\pi}^{\prime}, \boldsymbol{v}=\mathbf{0}$. 
If not, the last equation of the system (37) can be written as $\pi_{1}+\pi_{3}-2 \pi_{2}=0$, and its solution is defines with the expression:

$$
\begin{gathered}
\pi_{1}=\pi_{1}^{\prime}-\alpha_{1} v_{1}, \\
\pi_{2}=\pi_{2}^{\prime}+2 \alpha_{2} v_{1}, \\
\pi_{3}=\pi_{3}^{\prime}-\alpha_{3} v_{1}, \\
v_{1}=\frac{\pi_{1}^{\prime}+\pi_{1}^{\prime}-2 \pi_{2}^{\prime}}{a_{1}\left(\alpha_{1}+\alpha_{3}+4 \alpha_{2}\right)} .
\end{gathered}
$$

Clause 2.

If the vector of CI $\mathbf{y} \in \mathbf{R}^{\mathrm{M}}$ is centered to metric $\mathbf{A}$, then the vector $\boldsymbol{\kappa}=\mathbf{H} \boldsymbol{\pi}$

where $\pi$ - the solution of the problem (27), (28), is Acentered.

Proof.

The continued equality is valid:

$$
\mathbf{1}^{\prime} \mathbf{A} \boldsymbol{\pi}=\sum_{\mathrm{i}=1}^{\mathrm{M}} \mathrm{p}_{\mathrm{i}} \kappa_{\mathrm{i}}=\sum_{\mathrm{i}=1}^{\mathrm{M}} \mathrm{p}_{\mathrm{i}} \pi_{\mathrm{r}_{\mathrm{i}}}=\sum_{\mathrm{i}=1}^{\mathrm{N}} \pi_{\mathrm{i}} \sum_{\mathrm{j} \in \mathrm{N}_{\mathrm{i}}} \mathrm{p}_{\mathrm{j}}=\sum_{\mathrm{i}=1}^{\mathrm{N}} \mathrm{n}_{\mathrm{i}} \mathrm{p}_{\mathrm{i}}
$$

Since the sum of each column of matrix $\nabla \mathbf{g}(\boldsymbol{\pi}), \nabla \mathbf{h}(\boldsymbol{\pi})$ is equal to zero, when summing over all the equations of the system (30), we get

$$
\sum_{i=1}^{N} n_{i} \pi_{i}=\sum_{i=1}^{N} n_{i} \pi_{i}^{\prime}=\sum_{i=1}^{N} \sum_{j \in N_{i}} p_{j} y_{j}=\sum_{i=1}^{M} p_{j} y_{j}=0
$$

The affirmation of the clause follows from relations (38), (39).

\section{Theorem 2.}

The vector $\boldsymbol{\kappa} \in \mathbf{R}^{\mathrm{M}}$, built by the Algorithm 1 is centered and normalized in metrics Aby the solution of the problem (26).

Proof.

The problem (26) is equal to the problems 27), (28), and the conditions of the Kuhn-Tucker theorem for the latter can be written as:

$$
\begin{gathered}
\nabla_{\pi} \mathrm{L}(\boldsymbol{\pi}, \boldsymbol{\lambda}, \boldsymbol{\mu})=\mathbf{0} \\
\mu_{\mathrm{j}}\left(\pi_{\mathrm{j}}-\pi_{\mathrm{j}+1}\right)=0, \mathrm{j} \in \overline{1, \mathrm{~N}-1} ; \\
v_{\mathrm{k}} \mathrm{h}_{\mathrm{k}}(\boldsymbol{\pi})=0, \mathrm{k} \in \overline{1, \mathrm{~N}-2} ; \\
\|\mathbf{H} \boldsymbol{\pi}\|_{\mathbf{A}}^{2}-1=0 ; \\
\mu_{\mathrm{j}} \geq 0, \mathrm{j} \in \overline{1, \mathrm{~N}-1} ; v_{\mathrm{k}} \geq 0, \mathrm{k} \in \overline{1, \mathrm{~N}-2}
\end{gathered}
$$

Using the Algorithm 1, first let us calculate the vector $\boldsymbol{\pi}^{\prime \prime}$ according to the methods of solution of a system of equations (30), thus the vector $\boldsymbol{\pi}^{\prime \prime}$ along with the vectors $\boldsymbol{\mu}, \boldsymbol{v}$ are the solution of the system (30), that is the conditions (40)-(42) are met.
In order to obtain the corresponding solution $\mathbf{K}^{\prime \prime}=\mathbf{H} \boldsymbol{\pi}^{\prime \prime}$ of the problem (26), it is necessary to normalize $\boldsymbol{\kappa}^{\prime \prime}$, that is to take $\boldsymbol{\kappa}=\boldsymbol{\kappa}^{\prime \prime} /\left\|\boldsymbol{\kappa}^{\prime \prime}\right\|_{\mathrm{A}}$.

Hence, the condition (43) is met for the equivalent problem.

Therewith, the conclusion of the theorem implies that the problem (27), (28) is equivalent to (26).

\section{CONCLUSION}

As a result of the performed research, the optimal RI for CENS of MR has been synthesized using the nominal and hyperordinal scales. The algorithm of the synthesis of the optimal RI in the hyperordinal scale has been developed. It is confirmed that the vector of the optimal digitalization in the scales in question is centered and normalized in the metric A.

\section{REFERENCES}

1. V. Antyufeev. Matrix radiometric correlationextreme navigation systems for aircraft: monographUkraine, Kharkov: KhNU V.N. Karazin, 372 p.

2. A. Sotnikov, V. Tarshyn, N. Yeromina, S. Petrov, N. Antonenko. A method for localizing a referenceobject in a current image with several bright objects, EasternEuropean Journal of Enterprise Technologies. 2017. Vol. № 9 (87). pp. 68-74.

DOI: $10.15587 / 1729-4061.2017 .101920$.

3. N.Yeromina, S. Petrov, A. Tantsiura, M. Iasechko, V. Larin. Formation of reference images and decisionfunction in radiometric correlation-extremal navigation systems. Eastern-European Journal ofEnterprise Technologies. - 2018. Vol.4, No.9 (94). - pp. 27-35. DOI: 10.15587/1729-4061.2018.139723.

4. S. Trehubenko, L. Berkman, N.Yeromina, S. Petrov, Y. Bryzhatyi, H. Kovalov, V.Dachkovskyi, L.MikhailovaThe Operation of Detection Systems in Conditions of Contrast Decrease of Ground Objects, JETER, 8 (1), 2020, pp. 208-212,

doi: $10.30534 /$ ijeter/2020/28812020

5. N.Yeromina, S. Petrov, A. Tarasenko, N. Antonenko, O. Semenenko, O. Pavlovskyi, M. Sliusarenko, V. TelehinThe Use of Strong and Ordinal Scales during the Synthesis of Reference Images for Vehicle Correlation-Extreme IJATCSE, 9 (2), 2020, pp. 23432349, doi: 10.30534/ijatcse/2020/218922020

6. O.Tymochko, A.Ttystan , V.Ushan , N.Yeromina , O.Dmitriiev, V.Mazharov, I.Padalka, I.Hannoshyna, I. Masik , A. ZazirnyiThe Synthesis of the Reference Image and Algorithms for Vehicle Navigation Systems, JETER,8(3), 2020, pp. 853- 858.

doi: $10.30534 /$ ijeter/2020/40832020

7. Pfanzagl I. Measurement theory. - Moscow: Mir, 1976. - 248 p.

8. Grenander W. Lectures on the theory of images : TRANS.: In 3 t. - M.: Mir. Vol. 1: image Synthesis. 1979. $-383 \mathrm{p}$.

9. Gutkin L.S. Optimization of electronic devices by a set of quality indicators. - M .: Owls. Radio, 1975 .-$368 \mathrm{p}$.

10. Lancaster P. Theory of matrices: Per. from English. Moscow: Nauka, 1978, 202 p 